\title{
Lexis
}

Journal in English Lexicology

9 | 2015

Utterer-Centered Studies on Lexical Issues

\section{Construction d'un modèle combinatoire fondé sur l'analyse de la polysémie du verbe perdre}

\section{Philippe Planchon}

\section{CpenEdition}

\section{Journals}

\section{Édition électronique}

URL : http://journals.openedition.org/lexis/946

DOI : 10.4000/lexis.946

ISSN : 1951-6215

\section{Éditeur}

Université Jean Moulin - Lyon 3

Référence électronique

Philippe Planchon, «Construction d'un modèle combinatoire fondé sur l'analyse de la polysémie du verbe perdre », Lexis [En ligne], 9 | 2015, mis en ligne le 13 mai 2015, consulté le 01 mai 2019. URL: http://journals.openedition.org/lexis/946 ; DOI : 10.4000/lexis.946

Ce document a été généré automatiquement le 1 mai 2019.

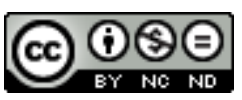

Lexis is licensed under a Creative Commons Attribution-NonCommercial-NoDerivatives 4.0 International License. 


\title{
Construction d'un modèle combinatoire fondé sur l'analyse de la polysémie du verbe perdre
}

\author{
Philippe Planchon
}

Je souhaite remercier les relecteurs de cet article pour leurs judicieuses remarques. Je remercie par ailleurs Jean Chuquet, Hélène Chuquet, Michel Paillard et Sarah de Vogüé, pour leurs nombreux conseils et encouragements.

\section{Introduction}

1 Un des principes fondateurs de la Théorie des Opérations Prédicatives et Enonciatives (TOPE) est le refus d'une séparation de principe entre les domaines de la sémantique, de la syntaxe et de la pragmatique [Culioli $2002: 39$ ]. L'application de ce principe au domaine de la sémantique lexicale justifie la mise en œuvre de l'hypothèse des formes schématiques pour l'étude de différents marqueurs polysémiques ${ }^{1}$.

2 Voici comment cette hypothèse a été formulée par A. Culioli :

L'analyse d'un marqueur (...) doit aboutir à une représentation formelle ayant des caractéristiques stables et contrôlables. A partir de cette représentation formelle, que j'appelle forme schématique, se constituent des formes supplémentaires qui sont, en fait des déformations de la forme de base. [Culioli 1990 : 115]

L'idée selon laquelle ce dispositif est à la fois stable et déformable s'adapte bien à l'analyse de la polysémie, si l'on n'entend pas réduire celle-ci à une forme de monosémie (absence de variation), ou d'homonymie (absence d'identité). Il reste toutefois à définir le statut à accorder au lexique dans une telle perspective.

Doit-on tenir le matériel lexical pour une composante essentielle ou accessoire dans l'analyse de l'énoncé, de son organisation sémantique, syntaxique et énonciative? 
5 L'attention portée aux faits lexicaux au cours des dernières décennies témoigne ici d'une évolution: "Initialement élaborée pour étudier le fonctionnement des marqueurs énonciatifs, la théorie tend actuellement à s'étendre aux faits de lexique, qu'elle avait pendant longtemps laissés dans l'ombre, et à rendre compte, avec le même corps de concepts, du fonctionnement de certains morphèmes lexicaux » [Fuchs $1992: 225]$.

6 L'alternative qui se présente à la TOPE a été formulée comme suit par J.-C. Milner : le matériel lexical tombe-t-il « sous la juridiction de la linguistique énonciative », ou doit-il être considéré comme « une sorte de vêtement externe (...) ne révélant rien de décisif sur l'organisation énonciative elle-même »? [Milner, 1992 : 32-33].

7 Cette question est essentielle si l'on ne souhaite pas reconduire une conception modulaire et classificatoire du lexique au sein même de la TOPE. En effet, une telle conception reviendrait à maintenir une séparation de principe entre le lexique et la syntaxe [De Vogüé 1989 : 36]. Il convient donc d'opposer à cette approche modulaire une approche intégrative et constructiviste: tout marqueur est marqueur d'opérations, et l'énoncé résulte de l'agencement de ces marqueurs. Leur éventuelle catégorisation ne peut opérer que s'il est tenu compte de leurs modalités d'intégration dans l'énoncé.

8 À cet égard, l'analyse des verbes polysémiques constitue un enjeu décisif pour le débat qui oppose ces deux conceptions. Le verbe est central dans l'organisation énonciative et syntaxique de l'énoncé, alors même qu'il s'agit d'un élément lexical.

9 Nous avons choisi de faire porter notre analyse sur le verbe perdre en français contemporain. En effet, ce verbe a fait l'objet de différentes études, en relation avec sa polysémie et son fonctionnement syntaxique. Il permet donc d'illustrer la problématique des relations entre les domaines du lexique, de la syntaxe et de la sémantique.

Nous proposons d'articuler la polysémie du verbe perdre à différents plans de variation, et de lui associer une forme schématique apte à en modéliser le fonctionnement. Nous souhaitons ainsi montrer l'intérêt d'une approche intégrative et constructiviste du lexique dans une perspective énonciative.

\section{La logique classificatoire}

Dans le domaine lexical, l'approche constructiviste conduit à passer d'une logique classificatoire à une logique des fonctionnements (De Vogüé [1991 : 52-54]).

12 L'étude de différents verbes a ainsi pu montrer qu'il n'était pas possible d'opérer une catégorisation des verbes dans le cadre des typologies de procès, dès lors qu'il était tenu compte de leur polysémie (Franckel et al. [1989]).

13 Il en va de même pour le verbe perdre: celui-ci semble échapper à toute tentative classificatoire, bien qu'il soit traditionnellement catégorisé comme verbe d'achèvement, dans la suite des classifications proposées par Kenny [1963] et Vendler [1967].

Les verbes d'achèvement se caractérisent par leur incompatibilité avec commencer, continuer à, finir de (Fuchs [1991: 11]). Or, ces constructions se rencontrent avec perdre :

(1) j'ai commencé très tôt à perdre mes cheveux (Jacques Roubaud, FT. R000) ${ }^{2}$

(2) Est-ce que nous allons continuer à perdre, une à une, toutes nos terres

d'Union française ? (Pierre Mendès-France, FT. S188)

(3) Elle était toujours alitée et finissait de perdre sa vie dans une pièce poussiéreuse et sans air. (Robert Sabatier, FT. R807) 
15 À la différence des verbes d'état, les verbes d'achèvement sont incompatibles avec cesser de. Par contre, ils ont en commun avec les verbes d'état d'être incompatibles avec être en train de. Or, perdre accepte ces deux constructions :

(4) Au cours de cette période, la monnaie n'a cessé de perdre de sa valeur. (Jean-Alain Lesourd, Claude Gérard, FT. P296)

(5) A cause de toi, il est en train de perdre le peu de foi qu'il avait en luimême. (François Mauriac, FT. L684)

Les verbes d'état sont par ailleurs incompatibles avec se mettre à et s'arrêter de. Or, ces constructions s'emploient également avec perdre:

(6) Si Dabek se mettait à perdre la tête, c'était fini... (René Fallet, FT. R538)

(7) Ils savent bien qu'ils n'ont échangé un néant que contre un autre, comme le joueur qui change les règles du jeu de hasard sans pouvoir s'arrêter de perdre. (Jean Grenier) ${ }^{3}$

Perdre n'est donc pas un verbe d'achèvement ni un verbe d'état. S'agit-il d'un verbe d'activité ou d'accomplissement? Les verbes d'activité se caractérisent tout d'abord par leur incompatibilité avec l'emploi d'un nom de durée dans la construction mettre $\mathrm{N}_{\text {durée }}$ à. Or, cette construction est compatible avec perdre:

(8) je reprends en une malheureuse journée ce que j'avais mis plusieurs jours à perdre $(700 \mathrm{~g})$. (http://forum.doctissimo.fr)

Par ailleurs, l'opposition entre activité et accomplissement se fonde sur une différence d'acceptabilité dans l'emploi de compléments de durée introduits par les prépositions en et pendant. Or, ces deux types de compléments se retrouvent employés avec perdre:

(9) Il perdit ainsi, en un seul jour, plusieurs centaines de millions. (Marcel Aymé, FT. R526)

(10) Il l'avait perdue de vue pendant plusieurs années. (Jean d'Ormesson, FT. S299)

Et il n'est pas possible d'intervertir ici ces prépositions :

(11) *Il perdit ainsi, pendant un seul jour, plusieurs centaines de millions.

(12) *Il l'avait perdue de vue en plusieurs années.

Perdre peut s'interpréter selon le cas comme verbe d'accomplissement (9) ou comme verbe d'activité (10). Les tests utilisés pour différencier les types de procès donnent des résultats variables selon le contexte. La polysémie de perdre exclut de le rattacher à un seul type de procès. En réalité, la catégorisation de perdre comme verbe d'achèvement ne fonctionne que si l'on ne retient qu'un seul de ses emplois (celui où il dénote une défaite) comme représentatif de ses propriétés aspectuelles. Il est donc préférable de dépasser cette logique classificatoire au profit d'une logique des fonctionnements.

\section{Les modes de fonctionnement}

21 Dans la perspective définie par la logique des fonctionnements, les différents emplois du verbe ont tous une même importance. La valeur de défaite illustrée par l'exemple (13) n'est donc qu'un cas particulier parmi d'autres : 
(13) Il a perdu le match.

(14) Il a perdu du sang.

(15) Il a perdu son temps.

La distinction entre ces trois modes de fonctionnement ne doit pas s'entendre comme une simple reformulation d'autres typologies. Comme l'indique S. de Vogüé [1987 : 60], cette distinction a surtout pour mérite de les rapporter à une problématique commune, qui est celle de la construction d'occurrences :

Autant dire que ce mérite est de ne pas s'en tenir au stade simplement descriptif classificatoire-: elle explique ce qui est à l'origine des différences de comportements entre ces types, et dès lors permet des prédictions [...]. De telles prédictions sont particulièrement importantes dans la mesure où un des problèmes essentiels qui se pose à la classification des procès en types est que les mêmes verbes puissent prendre un comportement totalement différent selon le contexte.

Suivant cette hypothèse, la distinction discret - dense - compact constitue un plan régulier de variation. La distinction entre ces modes de fonctionnement repose elle-même sur la distinction entre délimitation qualitative (Qlt) et délimitation quantitative (Qnt) des procès, et sur les relations entre la notion et les occurrences de procès (Culioli [1999b : 14-15] ; Franckel \& Paillard [1991]).

Dans le fonctionnement dense, l'occurrence de procès est associée à une classe d'instants (Qnt), et définie comme conforme à la notion de procès pour tout instant inclus dans cette classe d'instants. Quelle que soit la durée associée au procès, le procès est validé du simple fait qu'il est localisé dans le temps. Ainsi, dans l'exemple (14), le procès perte de sang est validé pour toute la durée du procès. Si le procès s'interrompt, il ne se trouve pas invalidé pour autant, et peut reprendre à tout instant.

Dans le fonctionnement discret, l'occurrence de procès est également localisée par une classe d'instants (Qnt), mais celle-ci est structurée par une borne qui en définit l'extensité, c'est-à-dire le temps mis pour atteindre l'état résultant (Qlt). Si le procès s'interrompt, il se trouve invalidé (depuis quelques minutes, il a cessé de perdre le match). Dans l'exemple (13), le statut de perdant implique l'achèvement du procès : le match n'est perdu qu'une fois terminé. Il y a donc une stricte complémentarité entre le déroulement du procès et l'état résultant (De Vogüé [1989 : 28]).

Dans le fonctionnement compact, le procès n'est pas validé en fonction de sa localisation dans le temps, bien qu'il soit toujours possible de le limiter à une certaine durée ${ }^{4}$. La validation du procès repose sur les propriétés qualitatives associées à la notion de procès : les entités et relations définies par l'énoncé sont reformulées selon cette notion (Qlt). Il n'y a pas d'occurrence de procès. L'exemple (15) est à cet égard significatif : il ne s'agit pas d'indiquer un prélèvement sur une durée disponible, ni de localiser un événement dans le temps, mais de qualifier sous forme de perte (comme relation détrimentale) la relation entre un sujet et la situation définie par l'énoncé.

Nous proposons de définir le fonctionnement général du verbe perdre comme la mise en relation d'une différence qualitative ou quantitative avec une différence entre deux points de vue, deux instants ou deux situations. Dans l'exemple (15), l'emploi du nom temps complique quelque peu l'analyse, mais il convient de remarquer qu'il s'agit là du 
nom le plus fréquemment employé ${ }^{5}$ comme complément d'objet direct $\left(\mathrm{C}_{1}\right)^{6} \mathrm{du}$ verbe perdre.

Cette construction est d'ailleurs intéressante, car il est possible de distinguer entre perdre son temps et perdre du temps, comme le montrent les exemples (16) et (17) :

(16) Tu comprends, j'ai préféré rentrer. C'était inutile que je reste là-bas. Je perdais mon temps. (Raymond Queneau, FT. R015)

(17) A cause de l'agitation, a dit Haymann, j'ai voulu aller voir. Mais j'avais perdu du temps. Quand je suis arrivé, c'était tout bouclé. (Jean-Patrick Manchette, FT. S567)

Dans l'exemple (16), il s'agit de qualifier le temps écoulé par rapport à une valeur de référence ou une norme de ce que serait le meilleur usage possible du temps qui passe, du point de vue de l'énonciateur. Cette différence d'évaluation s'articule alors sur une alternative entre deux situations (rester là-bas vs rentrer).

Par contraste, l'exemple (17) indique le prélèvement d'une quantité de temps sur le temps qui est disponible pour atteindre un objectif (j'ai voulu aller voir) : on retrouve alors un fonctionnement dense du verbe.

\section{Les contraintes d'interprétation sur l'énoncé}

La distinction entre modes de fonctionnement permet de donner une nouvelle interprétation aux tests mis en œuvre dans les exemples (1) à (12).

Parmi ces tests, nous nous limiterons ici à l'emploi de être en train de, avant d'aborder le passé composé et la négation.

Etre en train de est compatible avec les différents modes de fonctionnement du verbe. Mais son interprétation ne sera pas la même selon les cas :

(18) Elle interrompit un instant une partie d' « hombre » qu'elle était en train de perdre, n'ayant jamais pu apprendre les règles d'aucun jeu. (Françoise Chandernagor, FT. S386)

(19) l'un de nous deux est en train de perdre tout son sang. (Philippe Djian, FT. R813)

(20) J'ai eu peur. J'étais en train de perdre un ami. Ses yeux lançaient des foudres. (Romain Gary, FT. S524)

Selon Franckel [1989: 65 sqq.], être en train de permet de marquer une forme de discordance entre la délimitation qualitative du procès et son ancrage temporel.

Avec un fonctionnement discret (18), cette discordance tient à l'existence d'un moment à partir duquel le procès peut être validé, alors que l'énoncé se rapporte à un moment antérieur à cet instant et introduit donc une tension entre ce qui a lieu actuellement et l'état résultant envisagé comme issue probable du procès.

Avec un fonctionnement dense (19), la discordance consiste à articuler l'absence de borne intrinsèque d'un procès dense et son caractère temporaire. La différence avec le présent simple est en général très peu sensible. Elle est toutefois plus marquée dans l'exemple (19), par la présence du déterminant tout: le procès reçoit une borne extrinsèque qui renforce son caractère menaçant, et la nécessité de l'interrompre ${ }^{7}$.

Avec un fonctionnement compact (20), la discordance tient à la prise en compte d'un point de vue extérieur aux conditions de validation du procès, ce qui amène une valeur 
d'incrédulité ou de surprise. Dans le cas présent, ce point de vue se trouve dissocié de la qualification de la situation comme situation détrimentale: le risque de perdre un ami n'avait pas été envisagé.

On observe une corrélation entre le mode de fonctionnement et l'interprétation des marqueurs d'aspect et de temps. A vrai dire, cette variation affecte la plupart des formes du verbe. Elle s'applique également à l'interprétation du passé composé, dont on sait qu'il constitue un temps particulièrement ambigu en français (Cotte [1987]).

Suivant l'analyse de J.-J. Franckel [1989 : 91], le passé composé marque une opération de localisation temporelle du procès sur (au moins) un instant $t_{i}$ distinct de $t_{0}\left(t_{i} \neq t_{0}\right)$. Or, «le croisement de cette opération avec les différentes configurations discret, dense, compact va déboucher sur des valeurs différentes » (Franckel \& Paillard [1991:121]), à savoir une valeur d'état résultant avec le fonctionnement discret, comme dans l'exemple (21), une valeur d'aoriste du discours avec le dense (22), et une valeur de passé révolu avec le compact (23) :

(21) Ça y est, ils ont perdu la guerre.

(22) Hier, le patient a perdu du sang.

(23) J'ai perdu mon temps à cette soirée.

Cette variation dans le fonctionnement du verbe affecte également l'interprétation de la négation. Comme l'indique S. de Vogüé [1991 : 64] :

[Avec un fonctionnement compact], il s'agit de prédiquer une propriété, [et] on est pris dans une opposition entre la propriété et son contraire - ce qui n'aurait pas vraiment de sens dans le cas d'un fonctionnement discret (on est alors dans une problématique du vraiment conforme, ou du pas vraiment conforme) ou dense (on est alors dans le domaine de la seule opposition présence versus absence).

Considérons tout d'abord un exemple de fonctionnement compact :

(24) Il a perdu son sang-froid !

Cet énoncé permet de prédiquer certaines propriétés qualitatives sur l'entité correspondant au sujet du verbe $\left(\mathrm{C}_{0}\right)$, par exemple en termes de colère ou d'énervement. L'emploi de la négation revient à opérer une prédication sur la propriété contraire :

(25) Il n'a pas perdu son sang-froid $\approx$ il est resté calme.

41 En revanche, avec un fonctionnement dense, comme dans l'exemple (26), la négation s'interprétera comme une absence d'occurrence de perte:

(26) Ce patient n'a pas perdu de sang.

Cet exemple n'implique pas la validation de ce qui serait le contraire d'une telle perte ( recevoir du sang?). Il en va d'ailleurs de même dans l'exemple (27), pour lequel il existe pourtant un verbe antonyme approprié dans ce contexte (prendre du poids) :

(27) Il a perdu du poids.

Il n'est manifestement pas possible de conclure de la négation d'une perte de poids à l'assertion d'une prise de poids :

(28) Il n’a pas perdu de poids $\neq$ Il a pris du poids. 
Enfin, avec un fonctionnement discret, la négation s'interprétera comme un hiatus entre les conditions de validation et de localisation du procès, comme dans l'exemple (29):

(29) Il n'a pas perdu la guerre.

Cet exemple présente une certaine ambiguïté. Le hiatus peut en effet porter sur les conditions de validation du procès (30) ou sur ses conditions de localisation (31) :

(30) On a pu dire qu'Hitler n'avait pas perdu la guerre puisque certains des poisons sécrétés par le nazisme se sont répandus dans l'ensemble du monde. (Pierre Mendès-France, FT. S190)

(31) La France a perdu une bataille! Mais la France n'a pas perdu la guerre ! (Charles de Gaulle, FT. R005)

Dans l'exemple (30), on peut parler d'une lecture qualitative de la négation: il s'agit de dire que Hitler a en un certain sens gagné la guerre, qu'il ne l'a pas vraiment perdue. En revanche, l'exemple (31) impliquera une lecture temporelle de la négation: l'objet de l'énoncé est de dire que la guerre n'est pas encore finie, qu'elle peut encore être gagnée.

\section{Les paramètres de variation du verbe perdre}

Le procès est caractérisé par ses propriétés qualitatives, c'est-à-dire par ce qui permet de dire qu'il s'agit bien de perdre dans les circonstances définies par l'énoncé. Mais le procès est également défini par ses déterminations quantitatives, par sa relation à la classe d'instants qui permet de localiser ou de valider le procès.

Nous avons vu que dans le cas d'un fonctionnement discret, ces deux modes de délimitation étaient articulés, ce que l'on peut noter ( $\mathrm{qlt}_{\mathrm{j}} \mathrm{F}_{\mathrm{K}} \mathrm{t}_{\mathrm{j}}$ ). Des noms comme guerre, match ou procès, définissent à la fois la durée et les circonstances de la défaite, en particulier l'existence d'un perdant et d'un gagnant. Le $C_{1}$ fonctionne alors comme repère constructeur (Paillard [1992]): ce qui est en jeu et peut être perdu est défini indépendamment de l'inscription de l'occurrence de procès dans le temps.

Il en va de même s'il s'agit d'une mise, à laquelle sera associée une visée et un état résultant contraire à cette visée. Le $\mathrm{C}_{1}$ ne sert pas alors à définir les circonstances de la défaite, mais l'ampleur de ce qui aura été perdu :

(32) Il a perdu sa fortune au poker.

Dès lors qu'il est possible d'associer au $C_{1}$ une visée, ainsi que la possibilité d'un écart ou d'un échec par rapport à cette visée, un fonctionnement discret du verbe peut se mettre en place, comme dans l'exemple (33):

(33) Il a perdu la cible.

51 Dans tous ces cas, le $\mathrm{C}_{1}$ sert à définir une valeur de référence, que l'on notera qlt $_{\mathrm{i}}$. Perdre marque l'écart entre cette valeur de référence et l'état résultant du procès ( $\mathrm{qlt}_{\mathrm{j}} \neq \mathrm{qlt} \mathrm{i}_{\mathrm{i}}$ ). Si une issue favorable (qlt $\mathrm{t}_{\mathrm{i}}$ ) est définie comme enjeu ou objet d'une visée, perdre servira à indiquer que c'est une issue défavorable (qlt $t_{j}$ ) qui se trouvera stabilisée, à partir d'un instant $t_{j}$ distingué dans le temps comme borne d'accomplissement du procès $\left(t_{j} \neq t_{i}\right)$. 

caractérise le fonctionnement dense du verbe. Le $\mathrm{C}_{1}$ ne sert alors qu'à spécifier l'ampleur et la nature de la perte. Ce sont les repères spatio-temporels de l'énoncé qui constituent le repère constructeur du procès, ce qui amène une forme de contingence dans la localisation de l'événement. Ce qui est perdu est prélevé sur l'ensemble de ce qui est perdable.

On pourra ici distinguer différentes formes de quantification à partir d'une valeur de référence qnt $t_{\mathrm{i}}$, qu'il s'agisse d'une mesure (34), d'une quantité prélevée (35), ou d'une localisation de référence (36) :

(34) Il a perdu dix kilos.

(35) Le moteur a perdu de l'huile.

(36) Le moteur a perdu une vis.

L'écart opère par rapport à une valeur de référence, ce que l'on notera $\left(\mathrm{qnt}_{\mathrm{j}} \neq \mathrm{qnt}_{\mathrm{i}}\right)$. L'écart peut être lui-même de nature spatiale, par exemple sous la forme d'une distance qui s'accroît (37), ou de nature temporelle comme dans l'exemple (38) :

(37) Je l'ai perdu de vue dans la foule.

(38) Il a perdu une opportunité.

L'écart peut lui-même être plus ou moins limité dans le temps, selon l'autonomie de ce qui est perdu. La possibilité de retrouver ce qui a été perdu permet en effet de circonscrire l'occurrence de perte :

(39) Il a retrouvé le portefeuille qu'il avait perdu pendant plusieurs jours. relation de localisation relève d'une forme d'ingrédience (Jackiewicz [1996]) ${ }^{8}$ :

(40) Il a perdu un bras à la guerre.

Enfin, lorsque perdre présente un fonctionnement compact, il permet de qualifier de perte ce qui n'apparait comme tel que d'un certain point de vue, par un changement d'état ou par comparaison avec une valeur qualitative qui constitue un état de référence.

Il est donc possible d'associer deux valeurs qualitatives qlt $_{\mathrm{i}}$ et qlt $_{\mathrm{j}}$ pour définir cet écart. Le $C_{0}$ n'est pas agentif et n'est pas le repère d'un changement de localisation: le $C_{0}$ constitue le support de prédication de ces propriétés qualitatives.

Le $\mathrm{C}_{1}$ permet ici de définir une valeur qualitative de référence, qu'il s'agisse d'une valeur attendue (41), d'une propriété de l'objet (42) ou d'une évaluation subjective (43) :

(41) Il a perdu son sang-froid.

(42) Ce meuble a perdu sa couleur.

(43) Cette question a perdu de son importance.

61 Comment représenter l'enchaînement des opérations associées au verbe perdre? 
La variation entre délimitations qualitatives et quantitatives de l'écart nous conduit tout d'abord à adopter comme notation $\mathrm{qt}_{\mathrm{i}}$ et $\mathrm{qt}_{\mathrm{j}}$ (Paillard [1988: 101]). Ces valeurs renverront selon les cas à des valeurs qualitatives ( qlt $_{i}$ qlt $_{j}$ ) ou quantitatives (qnt ${ }_{i}$ qnt $_{j}$ ), c'est-à-dire à des propriétés, des positions, des états, issues ou modes de relation.

Le verbe perdre indique un écart entre $\mathrm{qt}_{\mathrm{i}}$ et $\mathrm{qt}_{\mathrm{j}}$.

- La valeur $\mathrm{qt}_{\mathrm{i}}$ correspond à une valeur de référence. Il peut s'agir d'une valeur attendue ou espérée, d'une valeur bénéfique pour un sujet, d'une propriété inhérente à une entité, ou d'un repère stable dans l'espace ou dans le temps.

- La valeur $\mathrm{qt}_{\mathrm{j}}$ est différenciée de $\mathrm{qt}_{\mathrm{i}}$ de façon significative, qu'il y ait ou non continuité entre ces deux valeurs. Dans l'absolu, cela signifie qu'il peut y avoir rupture entre ces valeurs, si elles ne sont pas - ou ne sont plus - repérables l'une par rapport à l'autre ${ }^{9}$. On notera que l'opération de négation est ici construite ${ }^{10}$ par différenciation $\left(\mathrm{qt}_{\mathrm{j}} \neq \mathrm{qt} \mathrm{t}_{\mathrm{i}}\right)$.

Cette différence entre $q t_{i}$ et $q t_{j}$ est elle-même rapportée à une différence entre deux moments, deux points de vue, ou deux situations. Les valeurs $\mathrm{qt}_{\mathrm{i}}$ et $\mathrm{qt}_{\mathrm{j}}$ peuvent ainsi être orientées dans le temps, ou être définies hors-temps, à partir d'un point de vue subjectif ou selon la situation qui est envisagée.

A titre de synthèse, nous proposons de caractériser le verbe perdre par une forme schématique qui permet d'articuler ces différentes modalités de repérage : perdre marque un écart entre deux valeurs qualitatives ou quantitatives $\mathrm{qt}_{\mathrm{i}}$ et $\mathrm{qt}_{\mathrm{j}}$. La valeur $\mathrm{qt}_{\mathrm{i}}$ constitue une valeur de référence, dont $\mathrm{qt}_{\mathrm{j}}$ est différenciée de façon significative, en fonction de deux situations, moments ou points de vue.

\section{Les propriétés de fonctionnement du $\mathrm{C}_{1}$}

La construction de l'écart implique de prendre en compte certaines propriétés des compléments du verbe, et notamment les propriétés associées au $C_{1}$. En effet, l'écart ne met en jeu que certaines déterminations associées au $C_{1}$ selon ses modalités de repérage par rapport au $C_{0}$. Autrement dit, tout n'est pas perdu, ou ne l'est pas de la même façon.

Ainsi, s'agissant d'un objet que l'on a perdu (perdre ses clés), perdre n'implique pas que l'objet ait diminué, ait cessé d'exister ou que ses qualités aient changé : le fonctionnement discret du nom combiné au fonctionnement dense du verbe impliquera un défaut de localisation. Si l'on ajoute alors une visée, par exemple avec le verbe réussir, le procès sera discrétisé :

(44) Le bûcheron a réussi à perdre les enfants dans la forêt.

Le fonctionnement dense de perdre est d'ailleurs compatible avec une valeur bénéfique, neutre ou détrimentale. A cet égard, on pourra comparer les exemples (45) à (47) ${ }^{11}$ :

(45) L'avion perd de l'altitude, la collision pourra être évitée.

(46) L'avion perd de l'altitude, je pense qu'on va bientôt atterrir.

(47) L'avion perd de l'altitude, le crash semble inévitable.

Par ailleurs, un fonctionnement dense du $\mathrm{C}_{1}$ pourra être associé à un fonctionnement discret du verbe, si l'agentivité associée au $C_{0}$ est elle-même engagée : 
(48) On nous lançait en pleine tourmente à une heure difficile entre toutes, l'ennemi avançant avec une résolution forcenée, nos troupes perdant du terrain... (Maurice Genevoix, FT. R937) les propriétés de fonctionnement de ses compléments. De ce point de vue, les catégorisations qui ont cours dans le domaine nominal gagnent à être réinterprétées.

Nous prendrons ici l'exemple de la distinction entre animé et inanimé. Cette distinction est insuffisante pour rendre compte des variations d'interprétation du verbe. L'emploi d'un nom animé humain comme $C_{1}$ n'aura pas toujours la même fonction, et son interprétation pourra varier selon le fonctionnement du verbe. involontaire (à la différence de (44)) :

(49) «Le petit Alain Otispifre a perdu son papa. Celui-ci est prié d'aller le réclamer à la buvette du stade !» (René Fallet, FT. R538)

Dans l'exemple (50), le $C_{1}$ s'interprète comme une mise ou un enjeu, ce qui ramène à un fonctionnement discret de perdre, et soulève la question de l'agentivité associée au $\mathrm{C}_{0}$ :

(50) Taxi-Joë devait raconter comment ce pauv'taré de commerçant N'Doula, après avoir bu trop de vin de palme, avait perdu sa femme au jeu! (Jean Vautrin, FT. R964)

Ces deux exemples s'opposent à l'exemple (51), qui indique une situation de deuil :

(51) [ma marraine] parla tout le temps de ses malheurs. Depuis la dernière fois (...) elle avait perdu son mari, sa fille aînée et Gildéus, son frère. (Joseph Zobel, FT. S647)

Cet emploi rapproche le verbe perdre de la catégorie des verbes d'état (Normand [1996]) et peut être analysé comme un fonctionnement compact : l'événement de décès définit un changement de situation, qui ne s'interprète lui-même comme perte qu'au regard de l'effet détrimental produit sur un sujet (c'est une perte pour ma marraine).

Enfin, l'exemple (52) remet en cause la caractérisation de l'entité dénotée par le $C_{1}$ comme client, et se rapproche de l'exemple (20) vu précédemment (perdre un ami) :

(52) - Et pourquoi est-ce ennuyeux, de me donner une cabine double? Parce que cela vous coûtera plus cher, et que cela me fera peut-être perdre un client. (François-Régis Bastide, FT. S306)

Le $\mathrm{C}_{1}$ renvoie à un mode de relation, qui peut s'analyser comme un fonctionnement prédicatif du nom : < ( ) être client de $(\mathrm{)})>$, ce que l'on notera $\mathrm{C}_{1}-\mathrm{Q}$ à la suite de Franckel \& Lebaud [1990: 218], par opposition à un fonctionnement non-prédicatif $\left(\mathrm{C}_{1}-\mathrm{X}\right)$.

Cette dernière distinction n'est pas limitée aux animés humains, et on pourra de même opposer les exemples (53) et (54):

(53) $\mathrm{C}_{1}$-X : Le sort de Rudolf, qui perd la main avec laquelle il avait juré fidélité au roi avant de perdre la vie, est interprété comme une punition divine. (Peter von Moos) ${ }^{12}$ 
(54) $C_{1}-Q$ : nous tirons quelques coups de fusil pour ne pas perdre la main.

(Michel Tournier, FT. S310) l'exemple (55) :

(55) Long développement sur certains chaudronniers qui risquent de perdre la main (sic) si on les transfère. (Pierre Mendès-France, FT. S188)

fonctionnement prédicatif se signale par certaines contraintes sur la détermination nominale. La perte d'une faculté (perdre la raison) sera ainsi marquée par l'article défini singulier ( ${ }^{*}$ perdre une raison, ${ }^{*}$ perdre des raisons) $)^{13}$. La non-manifestation momentanée d'une aptitude sera marquée par l'absence de déterminant (Guillaume [1975:241]) : perdre pied (prédicatif) s'opposera par exemple à perdre un pied (à la guerre) ${ }^{14}$.

81 Le nom prédicatif peut par ailleurs avoir un répondant verbal (raison/raisonner), ce qui permet de retrouver certaines analyses avancées sur l'emploi support du verbe perdre (Vivès [1984]). Il s'agit toutefois ici d'élargir l'analyse aux conditions mêmes d'interprétation du nom, et non de la restreindre à ce critère ou à la possibilité d'une paraphrase par ne plus avoir, qui conduirait à exclure perdre haleine (*haleiner), ou encore perdre la vie (*il n'a plus la vie).

82 Le fonctionnement prédicatif du nom nous semble un argument décisif en faveur d'une analyse sémantique prenant en compte l'agencement des relations prédicatives et les conditions d'intégration des compléments du verbe dans le scénario verbal.

\section{L'hypothèse des modes de contextualisation}

La logique des fonctionnements prend tout son sens si l'on distingue entre les déterminations impliquées par le verbe et celles que le verbe reçoit de son contexte. L'articulation entre le verbe et le contexte permet alors de distinguer différents modes de contextualisation, suivant l'analyse qui en a été proposée par D. Paillard [2000] et S. de Vogüé [2004]. Il est ainsi possible de distinguer trois modes de contextualisation :

- nouage : le nouage est défini par un frayage entre les scénarios propres au verbe et à ses compléments, de sorte que ces scénarios se trouvent directement articulés entre eux. Le scénario verbal se trouve déjà mis en place par le contexte du verbe.

- greffe : la greffe correspond à une interaction partielle entre le scénario du verbe et ce qui relève de son contexte. Il en résulte la préservation d'une forme d'autonomie de part et d'autre, selon le point de vue adopté.

- ajout: l'ajout (ou juxtaposition) correspond au plus faible niveau d'intrication entre le scénario du verbe et ses compléments. Les compléments du verbe jouent un rôle secondaire et sont réinterprétés à partir du scénario verbal lui-même.

Malgré leur caractère hypothétique et provisoire, ces formulations permettent de cerner ce qui constitue la spécificité de chaque mode de contextualisation. Nous proposons d'en donner une illustration pour chacun des modes de fonctionnement du verbe perdre, en nous limitant ici à considérer les relations existant entre le verbe et le $\mathrm{C}_{1}$. 


\subsection{Contextualisation du fonctionnement DISCRET} repère à l'occurrence de procès. Tous les instants localisant l'occurrence en assurent la validation. Le $_{1}$ spécifie ce qui a été perdu, sur l'ensemble de ce qui peut l'être. Il ne s'agit pas d'un repère de construction, car ce qui a été perdu n'a pas vocation à l'être. (59) nouage - dense : Il a perdu du sang.

Le mode de fonctionnement discret conduit à prendre pour repère ce qui peut être perdu. Les conditions de validation du procès reposent sur un enjeu qui sert de repère à l'occurrence de procès. L'ancrage du procès suppose alors de structurer la classe des instants localisant le procès, en prenant pour repère le moment où le procès est validé.

(56) nouage - discret : Il a perdu le match.

De façon minimaliste, on peut observer que le nom match suffit à définir une période de temps, elle-même bornée à droite. Or, l'issue du match est caractérisée par une valeur de référence (le gagnant). Ces conditions répondent aux exigences posées par le fonctionnement discret du verbe perdre $\left(\mathrm{qlt}_{\mathrm{j}} \neq \mathrm{qlt}_{\mathrm{i}}\right.$ 侮 $\mathrm{t}_{\mathrm{j}} \neq \mathrm{t}_{\mathrm{i}}$ ). D'autres noms s'adapteraient mal à ces exigences (*perdre un concert, * perdre une promenade, *perdre une éclipse), du fait des propriétés temporelles, aspectuelles ou qualitatives qui s'y attacheraient.

(57) greffe - discret : Il a perdu le rythme. qualitative elle-même variable. Il faut une co-variation (et par suite une visée) pour obtenir un scénario du type «Il suivait (jusqu'alors) le rythme » (voir De Vogüé \& Paillard [1997] sur le verbe suivre). Cet exemple requiert d'articuler en partie le scénario de perdre sur le scénario défini par rythme, en interprétant convenablement ce dernier (comme « rythme à suivre »).

(58) ajout - discret : Il a perdu sa reine.

Dans le contexte ordinaire d'une partie d'échecs, sa reine peut définir un enjeu, au même titre qu'un match que l'on jouerait, ou qu'un rythme que l'on chercherait à suivre. Toutefois, les déterminations associées à sa reine ne servent pas à définir un scénario de perte : les propriétés de forme, de couleur ou de taille qui caractérisent sa reine ne sont pas concernées. De même, il n'est pas vrai de dire que sa reine cesse d'être sa reine pour être celle de son adversaire, ou cesse d'être une reine (pour devenir un fou ou une tour...). L'interprétation du procès repose uniquement sur le scénario mis en place par le verbe.

\subsection{Contextualisation du fonctionnement DENSE}

Le mode de fonctionnement dense procède d'un repérage spatio-temporel qui sert de

90 Le sang perdu n'est perdu que dans la mesure où il change de localisation, et ce changement de localisation opère par rapport à une localisation de référence définie par celui qui perd du sang. Autrement dit, il y a perte de sang dès lors que du sang se trouve localisé autrement que par le terme qui lui sert de localisateur de référence. Le nom sang 
suffit à définir ce qui pourrait constituer un scénario de perte de sang. Le verbe emprunte le chemin qui se trouve défini par ailleurs par le $\mathrm{C}_{1}$ (frayage notionnel).

(60) greffe - dense : Il a perdu du poids.

91 A l'inverse du cas précédent, le nom poids ne dessine pas un scénario de perte, mais il implique une forme de variation qui est par ailleurs compatible avec ce scénario. La valeur de référence n'est pas stable comme dans le cas de sang: la valeur de référence est fonction du poids à un moment antérieur. Le verbe perdre requiert une comparaison entre deux moments distincts, afin de mettre en place un scénario de perte de poids. La différence entre qnt $_{\mathrm{i}}$ et qnt $_{\mathrm{j}}$ n'est interprétable qu'à partir de ce repérage temporel.

(61) ajout - dense : Il a perdu du temps.

Le nom temps ne définit pas un scénario de perte, et la durée concernée par cette perte de temps n'est pas elle-même rapportée à un moment ou à une autre valeur qui en serait le repère. Le scénario d'un écart se trouve ici directement construit par perdre, et nécessite de rapporter cette quantité de temps à un objectif poursuivi (par exemple un trajet à effectuer dans «Il a perdu du temps sur la route »). La nature de ce qui a été perdu est certes spécifiée par le $C_{1}$, mais le $C_{1}$ ne définit pas lui-même un scénario de perte.

\subsection{Contextualisation du fonctionnement COMPACT}

Le fonctionnement compact revient à prédiquer une propriété qualitative d'une entité qui en est le support, et qui possède ses propres déterminations quantitatives. Dans le cas de perdre, il s'agit de définir l'état du support $\left(\mathrm{C}_{0}\right)$ par un écart qualitatif lui-même rapporté à une valeur de référence $\left(\mathrm{qlt}_{\mathrm{j}} \neq \mathrm{qlt}_{\mathrm{i}}\right)$. Par commodité, nous prendrons ici des exemples où le $\mathrm{C}_{1}$ renvoie à un animé humain, bien que ce trait ne détermine pas un fonctionnement compact (cf. fonctionnement dense dans (49) et discret dans (50)).

(62) nouage - compact : Non, je ne le trahirai pas, je perdrais un ami!

Le $C_{1}$ indique l'ampleur de ce qui a été perdu, c'est-à-dire la valeur de référence (qlt ${ }_{i}$ ). Toutefois, il ne permet pas de définir qlt ${ }_{\mathrm{j}}$, puisqu'il ne s'agit pas de dire qu'il cesse d'être un grand homme, bien au contraire ! En effet, il s'agit là d'un nom qualifiant (Milner [1978]) : la qualité de grand homme n'est prédiquée que d'un certain point de vue (pour la France), à la différence de noms classifiants (comme ingénieur). L'adjectif intervient alors pour qualifier la perte : c'est une grande perte pour la France (voir De Vogüé \& Franckel [2002] sur l'adjectif grand). L'intégration du $\mathrm{C}_{1}$ dans le scénario verbal engage une logique de points de vue, et le $C_{1}$ n'intervient que partiellement dans ce scénario. 
(64) ajout - compact : Pierre a perdu sa femme, il est très affecté par ce deuil.

96 A la différence de (63), sa femme ne permet pas de définir l'ampleur de la perte, mais sert uniquement à identifier l'un des repères de la prédication opérée sur l'état de Pierre. Il ne s'agit donc pas d'un nom qualifiant (cf. (63)), ni même d'un nom prédicatif (cf. (62)). Sa femme ne définit aucun scénario de perte, et c'est sur le verbe que repose la mise en place des ingrédients nécessaires à l'interprétation d'un tel scénario.

La distinction entre modes de contextualisation constitue selon nous une hypothèse importante sur l'articulation entre le scénario du verbe et son contexte. D'un point de vue sémantique, certains éléments semblent ainsi caractériser en propre chacun de ces modes de contextualisation. C'est le cas pour la logique de points de vue liée à la greffe, ou la réinterprétation du $C_{1}$ rendue nécessaire pour l'interprétation de l'ajout. Dans le cas de perdre, la greffe peut alors s'interpréter comme une forme de bilan négatif, l'ajout correspondant à un coup du sort, et le nouage à l'expression de l'impuissance du sujet. Il conviendrait donc de détailler et de systématiser ces principes de variation pour analyser l'organisation même de l'énoncé et ses différents degrés d'intégration.

\section{La variation du répertoire syntaxique}

Dans son article consacré à la polysémie verbale, D. Paillard [2000] propose d'identifier le répertoire syntaxique comme un troisième plan de variation du verbe, à côté des modes de fonctionnement et des modes de contextualisation.

Nous proposons pour finir d'aborder cet aspect de la variation de perdre, en évoquant brièvement quelques configurations liées à sa valence verbale ${ }^{15}$.

Nous avons centré jusqu'à présent notre analyse sur la relation entre le verbe et le $C_{1}$. Qu'en est-il lorsque perdre se construit sans $C_{1}$, c'est-à-dire sous forme intransitive? Quelles sont les variations de valence verbale que l'on peut observer avec ce verbe?

On observe tout d'abord que la valeur de défaite permet un emploi du verbe sans $C_{1}$, comme dans l'exemple (65):

(65) Goetz: Qui vous dit que je souhaite perdre? (Il met les dés dans le cornet). (Jean-Paul Sartre, FT. R447)

Sur le modèle de l'analyse conduite par Lambrecht et Lemoine [1996:292] à propos de gagner (la partie), nous parlerons dans ce cas d'une ellipse du $\mathrm{C}_{1}$, ou plus exactement, de l'absence d'instanciation de l'argument but du verbe. Cette possibilité pourrait ici s'expliquer par une contextualisation par nouage du fonctionnement discret du verbe.

Ce fonctionnement préserve une part d'agentivité $d u C_{0}$, et correspond à un emploi inergatif du verbe, si l'on suit les différents tests mis en avant dans la littérature à ce sujet (impératif, emplois absolus, adverbes...) (Legendre [1989]).

104 On opposera alors à l'exemple (65) d'autres emplois, strictement intransitifs, dans lesquels le $\mathrm{C}_{0}$ constitue l'argument but de la relation ${ }^{16}$, par exemple :

(66) Cette femme a (beaucoup) perdu.

(67) Maintenant le diamant perd tous les jours, le Brésil nous en accable depuis la paix (Honoré de Balzac, FT. R706) 
(68) Je ne puis m'empêcher de croire que le misanthrope perd à la scène. (Julien Green, FT. K943) réduite, alors qu'elle était préservée dans (71) par la bifurcation marquée par la préposition à. D'autre part, le préjudice semble sans limite dans (72), alors qu'il était limité dans (71) à la comparaison des avantages et inconvénients associés à l'ambition.

(72) Être ambitieux le perdra.

(69) A l'usage des jeunes paysans, on pourrait très bien établir des casernes loin de toute ville. Il est vrai que les patrons des maisons de tolérance y perdraient. (Simone Weil, FT. R514)

(70) Mais tout comme le lecteur y perd à ne pas voir la représentation sur scène, le spectateur lui aussi y perd à ne pas lire le texte de la pièce. (Michèle Touret $)^{17}$ selon que le $\mathrm{C}_{0}$ instancie ou non la relation prédicative $<()$ voir la représentation $>$, ce qui amène une différence qualitative pour le terme support $\mathrm{X}$ auquel renvoie le $\mathrm{C}_{0}$.

Or, il est possible de prendre un tel prédicat comme point de départ de la relation, et d'obtenir une construction causative : «le fait de passer d'une structure intransitive à une structure transitive peut consister à rajouter un sujet ou bien à rajouter un objet " (De Vogüé $[2004: 164])^{18}$. A titre d'illustration, prenons les exemples (71) et (72) :

(71) Il y perdra à être ambitieux.

La nominalisation renforce ce phénomène :

(73) Son ambition le perdra.

Une comparaison avec (74) montre que c'est le $\mathrm{C}_{0}$, et non plus le $\mathrm{C}_{1}$, qui est ici prédicatif :

(74) Il perdra son ambition.

En suivant l'analyse de J.-J. Franckel [1994], on peut alors caractériser (73) par la présence d'un $C_{0}$ prédicatif $\left(C_{0}-Q\right)$, et (74) par la présence d'un $C_{1}$ prédicatif $\left(C_{1}-Q\right)$. Dans les deux 
cas, le complément «son ambition » peut être glosé par < ( ) être ambitieux > (le référent du déterminant possessif instancie ici la place vide de la relation prédicative).

Le propre du $\mathrm{C}_{0}$ prédicatif dans (73) est d'associer une cause à un effet détrimental qlt (affectant l'entité $\mathrm{X}$ définie par le $\mathrm{C}_{1}$ ). Cette cause est l'état d'ambition (sit ${ }_{\mathrm{j}}$ ) qui caractérise en propre cette entité: par comparaison, l'absence d'ambition $\left(\right.$ sit $\left._{\mathrm{i}}\right)$ aurait des conséquences beaucoup moins dommageables $\left(\mathrm{qlt}_{\mathrm{i}}\right)$. On pourra donc analyser (73) par l'enchaînement suivant : $\mathrm{C}_{0}-\mathrm{Q}\left(\mathrm{sit}_{\mathrm{j}} \neq \mathrm{sit}_{\mathrm{i}}\right)$ perdra X ( $\left.\mathrm{qlt}_{\mathrm{j}} \neq \mathrm{qlt}_{\mathrm{i}}\right)$.

115 Ce schéma causatif permet d'avoir comme $C_{0}-Q$ une prédication de propriété (être curieux dans (75)), ou une forme d'activité nominalisée (méditer dans (76)) :

(75) Alors, il n'avait pas résisté au désir de fouiller dans le portefeuille de Fragonard. Oui, il se le répétait souvent, que la curiosité le perdrait. (Raymond Guérin, FT. R767)

(76) la méditation abstraite de son art, la rigueur appliquée à la culture des roses, ne peuvent que perdre un poète. (Paul Valéry, FT. K556)

Ainsi que le montre C. Normand [1996: 73-74], le cas de figure le plus intéressant est constitué par l'emploi d'un nom d'animé humain dans cette position, comme dans (77) :

(77) Pourtant, un sentiment de malaise, dont il n'eût pu comprendre le sens que plus tard, lui indiquait, avec d'obscurs moyens, que l'avocat le perdait. La cour maudissait un avocat si médiocre. (Jean Genet, FT. S381)

117 Le C $_{0}$ (l'avocat) n'a pas ici la même interprétation que dans l'exemple (78) :

(78) L'avocat a perdu le procès en appel.

Le $\mathrm{C}_{0}$ dans (77) est prédicatif, alors qu'il est non prédicatif dans (78). La meilleure glose de (77) passerait ici par l'emploi du verbe avoir : «L'avoir comme avocat causait sa perte ", ou encore : « le fait que son avocat soit si médiocre causait sa perte».

119 Comme on le voit, il n'est pas tant question d'agentivité que de propriétés qualitatives dans le fonctionnement causatif de perdre. Cet emploi confirme l'intérêt d'une analyse du fonctionnement des noms employés comme compléments du verbe perdre, pour l'interprétation du scénario du verbe et de ses interactions en contexte.

\section{Conclusion}

Ces derniers exemples nous semblent une illustration du principe selon lequel il n'est pas possible d'opérer une analyse du lexique sans tenir compte de l'agencement des relations prédicatives et des paramètres constitutifs de la situation d'énonciation.

121 L'analyse de la polysémie du verbe perdre suppose ainsi de pouvoir distinguer entre différents plans de variation. Les relations entre ces plans de variation doivent être reconstituées avec autant de précision que possible, ce qui requiert notamment de faire porter l'analyse sur les déterminations associées aux différents compléments du verbe.

122 L'enjeu est d'élaborer un modèle combinatoire qui permette de reconstituer les opérations dont les marqueurs présents dans l'énoncé sont la trace. Pour aboutir à un modèle cohérent et unifié, l'hypothèse des formes schématiques doit être appliquée à un grand nombre de marqueurs (lexicaux, grammaticaux ou discursifs), afin de mieux définir les principes qui ordonnent leur variation et de tester leurs interactions. 
En ce sens, la sémantique lexicale ne constitue qu'une étape dans l'élaboration d'un modèle combinatoire. Mais il s'agit bien d'une étape essentielle, si l'on adopte une perspective constructiviste et intégrative de l'énoncé et de ses conditions d'énonciation.

\section{BIBLIOGRAPHIE}

BLINKENBERG Andreas, 1960, Le problème de la transitivité en français moderne. Essai syntacticosémantique, Copenhague : Det Kongelige Danske Videnskabernes Selskab.

BOLBJERG Alfred, 1977, «Quatre groupes de la conjugaison -re : craindre :rendre prendre :mettre », Revue romane, $\mathrm{n}^{\circ} \mathrm{XII}-1,2-13$.

CAMUS Rémi \& DE VOGÜE Sarah (dir.), 2004, Variation sémantique et syntaxique des unités lexicales : étude de six verbes français, Linx, $\mathrm{n}^{\circ} 50$.

CARON Bernard, « Remarques sur la diathèse causative (anglais, français, haoussa) ", in La transitivité. Domaine anglais, Saint-Etienne : Université de Saint-Etienne, 1987, p. 29-48.

COTTE Pierre, 1987, « Réflexions sur l'emploi des temps du passé en français et en anglais à la lumière de deux évolutions récentes du système verbal de l'anglais ", Contrastes, $n^{\circ}$ 14-15, p. 89-161.

CULIOLI Antoine, 1990, Pour une linguistique de l'énonciation. Tome 1 : Opérations et représentations, Paris/Gap : Ophrys.

CULIOLI Antoine, 1999a, Pour une linguistique de l'énonciation. Tome 2 : Formalisation et opérations de repérage, Paris/Gap : Ophrys.

CULIOLI Antoine, 1999b, Pour une linguistique de l'énonciation. Tome 3 : Domaine notionnel, Paris/Gap : Ophrys.

CULIOLI Antoine, 2002, Variations sur la linguistique, Paris : Klincksieck.

DESCLÉS Jean-Pierre \& GUENTCHÉVA Zlatka, 1998, « Causalité, Causativité, Transitivité », in KULIKOV L. \& VATER H. (eds), Typology of Verbal Categories, Tübingen : Max Niemeyer Verlag, 7-27. DE VOGÜÉ Sarah, 1987, « Aspect : construction d'occurrences », T.A. Informations. Revue Internationale de l'Application de l'Automatique au Langage, $\mathrm{n}^{\circ}$ 1, 47-61.

DE VOGÜÉ Sarah, 1989, « Discret, dense, compact. Les enjeux énonciatifs d'une typologie lexicale », in J.-J. FRANCKEL (ed.), La notion de prédicat, Université Paris 7, Collection ERA 642, 1-37.

DE VOGÜÉ Sarah, 1991, « La transitivité comme question théorique : querelle entre la Théorie des Positions de J.C. Milner et la Théorie des Opérations Prédicatives et Enonciatives d'A. Culioli », Linx $\mathrm{n}^{\circ} 24,37-65$.

DE VOGÜÉ Sarah, 2004, « Syntaxe, référence et identité du verbe filer », Linx n 50, 135-167.

DE VOGÜÉ Sarah \& FRANCKEL Jean-Jacques, 2002, « Identité et variation de l'adjectif grand », Langue française, $\mathrm{n}^{\circ} 133,28-41$. 
DE VOGÜÉ Sarah \& PAILLARD Denis, 1997, « Identité lexicale et hétérogénéité de la variation co-textuelle. Le cas de suivre », in GUIMIER Claude, MEJRI Salah \& VICTORRI Bernard (eds), Co-texte et calcul du sens, Caen : Presses Universitaires de Caen, 41-61.

FALL Khadiyatoulah \& BÉTOTÉ-AKWA Doumbé, 2002, Enonciation et forme du sens. Identité lexicale et variations sémantiques des mots manger, aimer et raison, Les Presses Universitaires de Laval / Presses Universitaires de Limoges.

FRANCKEL Jean-Jacques, 1989, Etude de quelques marqueurs aspectuels du français, Genève/Paris, Librairie Droz.

FRANCKEL Jean-Jacques, 1994, « Effets sur le sens des verbes et la structuration de la relation prédicative de l'alternance sujet-prédicatif/sujet non-prédicatif », Cahiers de praxématique, $\mathrm{n}^{\circ} 22$, 157-175.

FRANCKEL Jean-Jacques (ed.), 2002, Le lexique, entre identité et variation, Langue française, $\mathrm{n}^{\circ} 133$. FRANCKEL Jean-Jacques \& LEBAUD Daniel, Les figures du sujet, A propos des verbes de perception, sentiment, connaissance, Paris/Gap : Ophrys, 1990.

FRANCKEL Jean-Jacques \& PAILLARD Denis, « Discret - Dense - Compact : Vers une typologie opératoire », in FUCHS Catherine (ed.), Les typologies de procès, Paris : Klincksieck, 1991, 103-136. FRANCKEL Jean-Jacques \& PAILLARD Denis, Grammaire des prépositions, tome 1, Paris/Gap : Ophrys, 2007.

FRANCKEL Jean-Jacques, PAILLARD Denis \& DE VOGÜÉ Sarah, 1989, « Extension de la distinction discret, dense, compact au domaine verbal », in DAVID J. \& KLEIBER G. (eds.), Termes massifs et termes comptables, Paris : Klincksieck, 239-247.

FUCHS Catherine, 1991, « Les typologies de procès : un carrefour théorique interdisciplinaire ", in FUCHS Catherine (ed.), Les typologies de procès, Paris : Klincksieck, 9-17.

FUCHS Catherine, 1992, " De la grammaire anglaise à la paraphrase : un parcours énonciatif ", in La théorie d'Antoine Culioli. Ouvertures et incidences, Paris/Gap : Ophrys, 221-226.

FUCHS Catherine \& LÉONARD Anne-Marie, 1980, «Eléments pour une étude comparée du déterminant zéro en anglais et en français ", in culıol Antoine (ed.), Opérations de détermination : théorie et description, Université Paris 7, LLF, 1-47.

GUILLAUME Gustave, 1975 [1919], Le problème de l'article et sa solution dans la langue française, Paris/ Québec, Librairie Nizet/Presses Universitaires de Laval.

JACKIEWICZ Agata, 1996, « L'expression lexicale de la relation d'ingrédience (partie-tout) », Faits de langues, $\mathrm{n}^{\circ} 7,53-62$.

KEENAN Edward, 1984, " Semantic correlates of the ergative/absolutive distinction », Linguistics, $\mathrm{n}$ - 22-2, 197-223.

KENNY Anthony, 1994 [1983], Action, Emotion and Will, Bristol : Thoemmes Press.

LAMBRECHT Knud \& LEMOINE Kevin, 1996, «Vers une grammaire des compléments zéro en français parlé ", in CHUQUET Jean \& FRYD Marc, Absence de marques et représentation de l'absence 1, Travaux linguistiques du Cerlico 9, Rennes : Presses Universitaires de Rennes, 279-309.

LEGENDRE Géraldine, 1989, “Unaccusativity in French”, Lingua, nº 79, 95-164. MILNER Jean-Claude, 1978, De la syntaxe à l'interprétation, Paris, Editions du Seuil. 
MILNER Jean-Claude, 1992, « De quelques aspects de la théorie d'Antoine Culioli projetés dans un espace non énonciatif », in La théorie d'Antoine Culioli. Ouvertures et incidences, Paris/Gap : Ophrys, 19-38.

NORMAND Claudine, 1996, «Perdre, quand dire c'est faire comme si », in costantini M. \& DarRault-harris I. (eds.), Sémiotique, Phénoménologie, Discours, Paris : L'Harmattan, 69-79.

PAILlARD Denis, 1988, «Temps, Aspect, Types de procès. A propos du présent simple ", in MILNER J.-C., Recherches nouvelles sur le langage, Université Paris 7, Collection ERA 642, 92-107.

PAILLARD Denis, 1992, «Repérage : construction et spécification ", in La théorie d'Antoine Culioli, Ouvertures et incidences, Paris/Gap : Ophrys, 75-88.

PAILlARD Denis, 2000, «A propos des verbes "polysémiques" : identité sémantique et principes de variation », in Sémantique du lexique verbal 2, Caen : Universitaires de Caen, 99-120.

PLANCHON Philippe, 2013, Etude de l'identité lexicale et de la variation sémantique des verbes accorder, donner et perdre en français contemporain, thèse de doctorat soutenue à l'Université de Poitiers le 16 janvier 2013.

PLANCHON Philippe, 2014, « Contraintes de modélisation de la polysémie du verbe perdre et de ses conditions d'interprétation en contexte ", Actes des $16^{\text {èmes }}$ Rencontres Jeunes Chercheurs en Sciences du Langage : Modèles et modélisation dans les sciences du langage, article publié en ligne : http:// hal.archives-ouvertes.fr/RJC2013, 13-23.

VENDLER Zeno, 1967, Linguistics in philosophy, New York : Cornell University Press.

VIVÈs Robert, 1984, «Perdre, extension aspectuelle du verbe support avoir », Revue québécoise de linguistique, $\mathrm{n}^{\circ} 13.2,13-57$.

\section{NOTES}

1. Voir le $n^{\circ} 133$ de Langue française (Franckel [2002]) et le $n^{\circ} 50$ de Linx (Camus \& De Vogüé [2004]). On pourra aussi se reporter à Franckel \& Lebaud [1990], Paillard [2000], et à Franckel \& Paillard [2007].

2. Parmi les exemples que nous proposons, les exemples attestés sont suivis du nom de l'auteur ou de l'URL s'agissant d'exemples trouvés sur Internet (dernière consultation le 01/12/13). Par défaut, les exemples sans référence ne sont donc pas des exemples de corpus, mais des exemples manipulés ou jugés énonçables. On notera pour finir que les exemples extraits de la base de données Frantext (www.frantext.fr) sont ici signalés par l'abréviation FT, suivie de la cote éditoriale employée dans Frantext pour référer aux textes du corpus.

3. Grenier Jean, Inspirations méditerranéennes, Paris, Gallimard, 1998 : 37.

4. Le caractère non-sécable du fonctionnement compact «bloque toute forme de délimitation notionnelle relayée sur le plan temporel, mais n'interdit pas une délimitation purement temporelle (cf. il a été méchant ce matin, il a été désagréable pendant tout le voyage) 》 (Paillard [1988 : 102]).

5. Nous présentons ailleurs des données statistiques sur le fonctionnement du verbe perdre (Planchon [2014]).

6. Nous utiliserons la convention proposée par Culioli [1999a : 26] pour noter les compléments du verbe selon leur ordre d'apparition, par exemple $C_{0}$ (sujet syntaxique), $C_{1}$ (complément d'objet direct)...

7. La présence d'une borne extrinsèque peut favoriser la discrétisation du procès. Toutefois, cela tient au mode de détermination $\mathrm{du} \mathrm{C}_{1}$, et non pas au mode de fonctionnement du verbe, qui 
relève ici d'un fonctionnement dense. Le raisonnement est en effet le même avec le partitif (l'un de nous deux est en train de perdre du sang). On remarquera d'ailleurs qu'il en va de même pour l'exemple (20), où la quantification sur ami (si l'on compare perdre un ami avec j'étais en train de perdre des amis) n'affecte pas les conditions de validation du procès, qui restent chaque fois caractéristiques d'un mode de fonctionnement compact.

8. La relation d'ingrédience peut alors être reformulée comme localisation préconstruite par rapport à un sujet. Cette forme de localisation subjective concerne notamment les parties du corps humain, mais peut s'appliquer tout aussi bien à une entité (inanimée) conçue comme un tout composé de parties (Le toit a perdu des tuiles).

9. Cette possibilité d'interpréter la différenciation comme rupture explique l'articulation spécifique des notions de continuité et de discontinuité dans le fonctionnement de perdre (Bolbjerg [1977: 6]), ainsi que certaines valeurs qu'il peut recevoir en contexte (perte réversible vs irréversible, perte progressive vs subite...).

10. «[Q]ue l'on considère la négation issue d'une opération de parcours avec construction de la partie vide de la classe d'occurrences, on retrouve d'un côté la négation qualitative de rejet par le sujet de ce qui est mauvais, de l'autre la négation de localisation et d'existence [...] l'opération de négation est primitive et, par complexification, se développe une négation construite à partir d'opérations telles que parcours, coupure, différenciation, inversion du gradient, sortie hors du validable » (Culioli [1990: 95]).

11. On peut noter ici que la valeur bénéfique illustrée par (45) est elle-même indépendante de toute visée, puisque l'exemple reste identiquement interprétable, que la perte d'altitude soit volontaire ou simplement fortuite.

12. Von Moos Peter, Entre histoire et littérature: communication et culture au Moyen Âge, Firenze, Edizioni del Galluzzo, 2005 : 166.

13. On pourra se reporter à la forme schématique du nom raison proposée par Fall \& Bétoté-Akwa [2002].

14. Pour une analyse de la détermination zéro conduite dans le cadre de la TOPE, voir notamment l'article de Fuchs \& Léonard [1980].

15. A cet égard, on notera que Blinkenberg propose de classer le verbe perdre parmi les verbes qui présentent une «bivalence en équilibre » [1960:115].

16. Ces emplois peuvent ainsi s'analyser par la distinction entre ergatif et absolutif (Keenan [1984]), qui articule la question de la valence verbale et l'hypothèse inaccusative (Legendre [1989]).

17. Touret Michèle, Lectures de Beckett, Presses Universitaires de Rennes, $1998: 43$.

18. Par ailleurs, l'ordre de dérivation peut opérer à partir de la structure intransitive ou de la structure transitive. Sur cette problématique et les relations entre transitivité et causativité, voir également Caron [1987], Desclés \& Guentchéva [1998].

\section{RÉSUMÉS}

En tant qu'approche centrée sur l'énonciation, la Théorie des Opérations Prédicatives et Enonciatives de Culioli peut difficilement faire l'économie d'une analyse des marqueurs linguistiques. Parmi ceux-ci, les unités polysémiques constituent un terrain privilégié d'analyse. A titre d'exemple, nous prendrons le verbe perdre en français, en nous appuyant sur des données 
de Frantext. Pour rendre compte du fonctionnement de ce verbe, nous distinguerons différents niveaux de variation, permettant de définir la forme schématique de perdre. L'articulation de ces différents niveaux et paramètres de variation permet de modéliser l'interaction entre le verbe et son contexte, et de définir les exigences d'une combinatoire en prise avec les paramètres de l'énonciation.

A speaker-centered theory, such as the Theory of the Predicative and Enunciative Operations of Culioli, cannot neglect the analysis of specific linguistic markers. In this regard, polysemous markers provide a rich field of study. This is why we have chosen to study the verb perdre in French, through data taken from the Frantext corpus. In order to understand the factors explaining how this verb interacts with its context and the enunciative situation, we need to distinguish between several levels of variation, from which it will be possible to define the schematic form of perdre. The modelling of these parameters will serve to outline the theoretical requirements of a combinatorial analysis.

\section{INDEX}

Keywords : schematic form, constructivism, context, polysemy, enunciation

Mots-clés : forme schématique, constructivisme, contexte, polysémie, énonciation

\section{AUTEUR}

\section{PHILIPPE PLANCHON}

Université de Poitiers, Laboratoire FoReLL - EA 3816

planchonphilippe@yahoo.fr 